\title{
Minimally invasive optical sensors for microwave- electric-field exposure measurements
}

\author{
Florent Behague, ${ }^{\text {a }}$ Venancio Calero, ${ }^{\text {a }}$ Antoine Coste $\odot,{ }^{\text {a }}$ Adrien Godet, ${ }^{\text {a }}$ \\ Miguel Suarez, ${ }^{a}$ Gwenaël Gaborit $\odot,{ }^{b}$ Lionel Duvillaret, ${ }^{b}$ Fadi I. Baida, \\ Maria-Pilar Bernal, ${ }^{b}$ and Nadège Courjal $\oplus^{a}$ * \\ ${ }^{a}$ Université Bourgogne Franche-Comté, Intitut FEMTO-ST, Besançon, France \\ ${ }^{\mathrm{b}}$ Kapteos SAS, Sainte-Hélène-du-Lac, France
}

\begin{abstract}
The measurement of microwave electric-field ( $E$-field) exposure is an ever-evolving subject that has recently led the International Commission on Non-Ionizing Radiation Protection to change its recommendations. With frequencies increasing toward terahertz (THz), stimulated by $5 \mathrm{G}$ deployment, the measurement specifications reveal ever more demanding challenges in terms of bandwidth (BW) and miniaturization. We propose a focus on minimally invasive $E$-field sensors, which are crucial for the in situ and near-field characterization of $E$-fields both in harsh environments such as plasmas and in the vicinity of emitters. We browse the large varieties of measurement devices, among which the electro-optic (EO) probes stand out for their potential of high BW up to THz, minimal invasiveness, and ability of vector measurements. We describe and compare the three main categories of EO sensors, from bulk systems to nanoprobes. First, we show how bulk-sensors have evolved toward attractive fibered systems that are advantageously employed in plasmas, resonance magnetic imagings chambers or for radiation-pattern imaging up to $\mathrm{THz}$ frequencies. Then we describe how the integration of waveguides helps to gain robustness, lateral resolution, and sensitivity. The third part is dedicated to the ultra-miniaturization of components allowing ultimate steps toward electromagnetic invisibility. This review aims at pointing out the recent evolutions over the past 10 years, with a highlight on the specificities of each photonic architecture. It also shows the way to future multi-physics and multi-arrays smart sensing platforms. (C) The Authors. Published by SPIE under a Creative Commons Attribution 4.0 Unported License. Distribution or reproduction of this work in whole or in part requires full attribution of the original publication, including its DOI. [DOI: 10.1117/1.JOM.1.2.020902]
\end{abstract}

Keywords: $E$-field sensors; photonics; electro-optics; radiofrequencies; microwaves.

Paper 20020V received Nov. 30, 2020; accepted for publication Mar. 8, 2021; published online Apr. 7, 2021.

\section{Introduction}

Exposure to microwave $E$-fields has been dangerously increasing with the growing wireless telecommunications, boosted by $5 \mathrm{G}$ deployment, the Internet of Things, and automotive vehicles. Apart from telecommunications, numerous applications are also concerned, such as biomedical with the expansion of e-health, ultra-high-field resonance magnetic imaging (RMI), plasmas, or radiofrequency treatments. The $E$-field omnipresence and the rise in frequencies induce new health and safety issues, which have pushed the International Commission on Non-Ionizing Radiation Protection to update its exposure guidelines. ${ }^{1}$ In this context, $E$-field measurement is more than ever required, be it for the evaluation of specific absorption rates (SARs), power density, ${ }^{2,3}$ or for assessing the emitted radiating fields. ${ }^{4}$ In this paper, we focus on the evaluation of $E$-fields emitted either in biomedical chambers such as magnetic resonance imaging (MRI) or cold plasmas, or in free space due to antennas or electrical devices. These applications are faced with ever-demanding specifications due to the frequencies increase toward terahertz (THz).

A first specification is to cover the entire emission spectrum, meaning more than 12 frequency decades for THz-emitting devices. As a second specification, the sensor should tend to minimal invasiveness, with a footprint far below the microwave wavelength. This feature

\footnotetext{
*Address all correspondence to Nadège Courjal, nadege.courjal@femto-st.fr
} 
is essential for plasma environments and near-field characterizations in the vicinity of emitters. Consequently, the footprint requires downscaling to submilliter as frequencies increase to $\mathrm{THz}$, and metals should be avoided. On the other hand, the probe compactness should not impact sensitivity, the latter being ideally below $10^{-2} \mathrm{~V} \mathrm{~m}^{-1} \mathrm{~Hz}^{-1 / 2}$. Third, the location of hot-spot requires micrometric spatial resolution.

One objective of electric field characterization is to detect harmful exposures to people or devices. Therefore, the fourth specification requires measuring the $E$-field vectors. Indeed, damages on living tissues and devices are known to depend on polarization. ${ }^{5,6}$ Linearity over large dynamic ranges facilitates calibration and reliable measurements and is thereby also a specification of interest. Finally, temperature stability stands as a must to avoid cumbersome and expensive temperature-control systems.

Although several technologies are now well established for electric field detection, they are struggling to meet these evolving requirements.

The most widely used $E$-field sensors are still metallic antennas. They are now remoted from the measurement system by an optical link to avoid radiation and cable interference and cover up to nine frequency decades. ${ }^{7}$ One way to perform this remote optical detection is via a photoconductive antenna. ${ }^{8}$ However, their intrinsic metallic composition and their centimeter footprint remain prohibitive for non-perturbative measurements.

Active sensors, which modulate a laser diode or a light-emitting diode to convert the electric signal into an optical signal, can provide $\mathrm{GHz}$ bandwidth (BW) with a millimeter footprint. ${ }^{8-10}$ But such active components are sensitive to magnetic-fields-induced perturbations. In addition, the active probe generates significant $E$-field perturbation.

Optical nanoantennas have emerged over the past decade as excellent candidates for minimally invasive measurements, ${ }^{11-13}$ leading to the in vivo study of electro-physiological fields. Their submicrometric footprint permits a long-term in vivo evaluation of the electric field impact onto the organism with nanometric spatial resolution. ${ }^{12}$ However, photonic nanoantennas rely on absorption measurements and cannot give direct information about the vectorial nature of fields.

Another approach based on an $E$-field-induced change of absorption but handling the problem of vector and phase measurement relies on Rydberg atoms. ${ }^{14-16}$ Due to their very high principal quantum number $n$, Rydberg atoms exhibit loosely bound valence electrons that can be easily perturbed by external fields, enabling ultra-enhanced sensitivity ${ }^{17}$ down to $55 \mathrm{nV} \mathrm{cm}^{-1} \mathrm{~Hz}^{-1 / 2}$ and absolute measurement of the $E$-fields. ${ }^{18}$ However, only narrow BW measurements (lower than $\mathrm{GHz}$ ) are easily feasible, and the method requires highly resolved spectroscopy.

Since the 1960s and the development of the first electro-optical (EO) $E$-field sensors, ${ }^{19,20}$ Pockels-based probes have experienced great success due to their intrinsic wide BW extending from DC to several $\mathrm{THz},{ }^{21,22}$ their associated subpicosecond temporal resolution, ${ }^{23}$ and their dielectric - and therefore minimally invasive-nature. By inducing a linear variation of the refractive index as a function of electric field components, the Pockels effect offers the additional advantage of enabling vector measurements, even in the most severe environments such as plasmas, ${ }^{24,25}$ lightning, ${ }^{26}$ gas insulation substations, or ultra-high voltage transmission towers. ${ }^{27}$ In the past, several comprehensive studies of Pockels-based probes have illustrated the attractive properties of EO sensors. ${ }^{27-30}$ But although the last decade has witnessed substantial evolutions in EO sensors, there is no review synthesising them. In this paper, we detail the developments in EO-based $E$-field sensors performed over the last decade. The new photonic EO architectures are highlighted and compared with the established EO configurations.

\section{Bulk Electro-Optic Sensors}

Pockels-based sensors, also called EO sensors, have been the object of growing attention for more than four decades. In their initial stage, ${ }^{20}$ the Pockels-based sensors relied on separated free-space optical elements, as represented in Fig. 1. In the classical configuration, which acts as a polarization state modulator (PSM), an EO crystal is placed between two crossed polarizers, and the linear polarization of the incident light is oriented $45 \mathrm{deg}$ to the crystal eigenvectors. The electrically induced linear birefringence generates a polarization change through the crystal, which is converted into intensity modulation by the second polarizer. A quarter-wave plate 


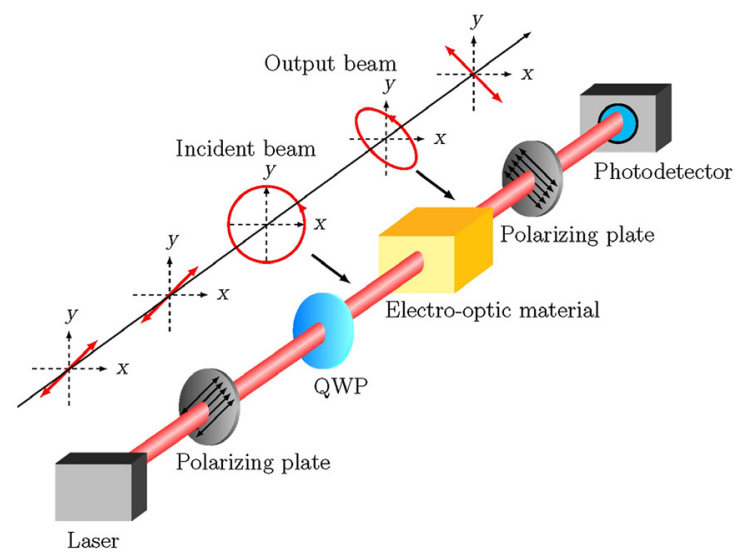

(a)

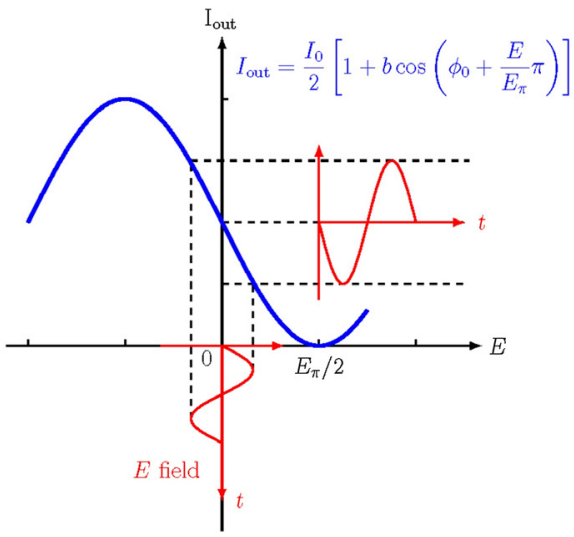

(b)

Fig. 1 Basic configuration for electric field sensing with a bulk EO crystal: (a) schematic view of the configuration and (b) EO response of the sensor. $I_{\text {out }}$ is the output irradiance. $E$ is the applied electric field.

is placed between the first polarizer and the EO crystal so that half of the light power passes through the system in the absence of electric fields. The small-signal response shows hence a linear sensitivity toward the $E$-field components [see Fig. 1(b)]..$^{30}$

When the optical probe is a pulse-delayed or chirped laser, the system can be used for EO sampling, leading to the measurement/recording of electric fields with frequencies up to $230 \mathrm{THz} .{ }^{22}$ Another particularity of free-space EO systems is their ability to provide very high spatial resolution by combining the proper choice of free-space imaging optics and an ultra-thin EO crystal. For example, a resolution as high as $\lambda / 600$ was reached by Blanchard and Tanaka ${ }^{31}$ with a $1-\mu \mathrm{m}$-thick $\mathrm{LiNbO}_{3}$ crystal. Another advantage of the free-space Pockels systems is the possibility to combine them with fast Mueller polarimetry. With this approach, Slikboer et al. ${ }^{32,33}$ recently showed the recovery of both the photoelastic and EO changes inside the sensing crystal, to obtain thereby a simultaneous time-resolved measurement of temperature and electric fields inside jet plasmas.

In the early 2000s, several institutions developed fibered versions of the PSM-based sensors, ${ }^{29,30,34,35}$ evolving thereby toward easier-to-use and less-invasive plug-and-play probes. In this case, a polarization-maintaining (PM) fiber is optically coupled to the EO crystal. Depending on the crystal thickness $t$, the crystal is either directly fixed to the bare fiber if $t<1 \mathrm{~mm},{ }^{36,37}$ or collimated with an intermediary GRIN fiber if $t>1 \mathrm{~mm} \cdot{ }^{25,27,35}$ The response is usually collected by reflection with a mirror (or a reflective coating such as distributed Bragg reflector $^{38}$ ) on the crystal backside. The reflected optical signal is collected back into a second fiber thanks to a slightly tilted incident angle to avoid return-light fluctuations. The quadrature bias is achieved similarly as in free space by placing a quarter-wave plate at the system input. ${ }^{25,27}$ To cancel out the potential effects of polarization fluctuations inside the PM fiber, Togo et al. ${ }^{39}$ used a Faraday rotator within the optical head, deleting the phase difference between the light transmitted on the fast and slow axes of the PM fiber. Compared to initial fibered systems, ${ }^{40}$ thin polarizers (100 $\mu \mathrm{m}$ or less) can now be inserted inside the probe head to lower the impacts of polarization instabilities.

Fibered EO-based bulk sensors are declined in various materials: an overview of their EO sensitivity is provided in Refs. 34, 41, and 42. Organic crystals usually show the highest EO coefficients, as for example, DAST with $r_{\mathrm{EO}}=52 \mathrm{pm} / \mathrm{V},{ }^{43} \mathrm{JRD} 1$ with $r_{\mathrm{EO}}=63 \mathrm{pm} / \mathrm{V},{ }^{44}$ SEO125 with $r_{\mathrm{EO}}=125 \mathrm{pm} / \mathrm{V},{ }^{45}$ or AJTB203 chromophores with $r_{\mathrm{EO}}=160 \mathrm{pm} / \mathrm{V}$. $^{46}$ However, polymer-based $E$-field sensors still lack stability compared to their crystalline counterparts. $\mathrm{LiNbO}_{3}$ or $\mathrm{LiTaO}_{3}$ also exhibits a high $r_{33} \mathrm{EO}$ coefficient: $r_{33 \text { _LiNbO3 }}=30.8 \mathrm{pm} / \mathrm{V}$ at $632.8 \mathrm{~nm} .{ }^{41}$ However, their temperature-dependent intrinsic birefringence requires stabilization by an external servo-controlled system. Though potentially useful as a temperature sensor, ${ }^{40,47}$ this additional control system also implies additional complexity and cost for $E$-field detection. Therefore, despite their lower EO efficiency, birefringent-free crystals such as BSO, ZnTe, or 
CdTe are appreciated for realizing temperature-stabilized measurements. ${ }^{39,48,49}$ The issue of the material's electromagnetic impedance matching is addressed with a proper encapsulation using matching layers. ${ }^{48}$ Another option to circumvent the impedance mismatch issue consists in detecting the $E$-field component tangential to the probe. Thereby, the transmitted field is not affected by the impedance mismatch due to the continuity equations. The technology is now mature for commercialization, as evidenced by Refs. 28, 50, and 51, and has become a dedicated technique in severe environments such as plasmas, ${ }^{24,25}$ lightnings, ${ }^{26}$ or MRI. ${ }^{52,53}$ PSM-based sensors are also ever-attractive for the near-field characterization of antennas, ${ }^{38,48}$ due to their minimal invasiveness associated with their wide BW and ability to perform vectorial measurements. The far-field can be extracted from the near-field mapping of the amplitudes and phases using the plane wave spectrum method, as illustrated in Fig. 2 for a quadridge horn antenna at a frequency of $30 \mathrm{GHz}$. Compared to its metallic counterparts, the PSM-based EO probe releases the need in cumbersome outdoor far-field installations or expensive anechoic chambers while also covering more than nine frequency decades. The performances of the PSM-based sensor used for Fig. 2 are shown in row \#3 of Table 1. The probe is made of BSO; the BW extends from $30 \mathrm{~Hz}$ up to $60 \mathrm{GHz}$, with an $E$-field sensitivity of $40 \mathrm{mV} \mathrm{m}^{-1} \mathrm{~Hz}^{-1 / 2}$ and a footprint of several $\mathrm{mm}^{3}$. In summary, PSM-based sensors represent a good compromise between BW, footprint,

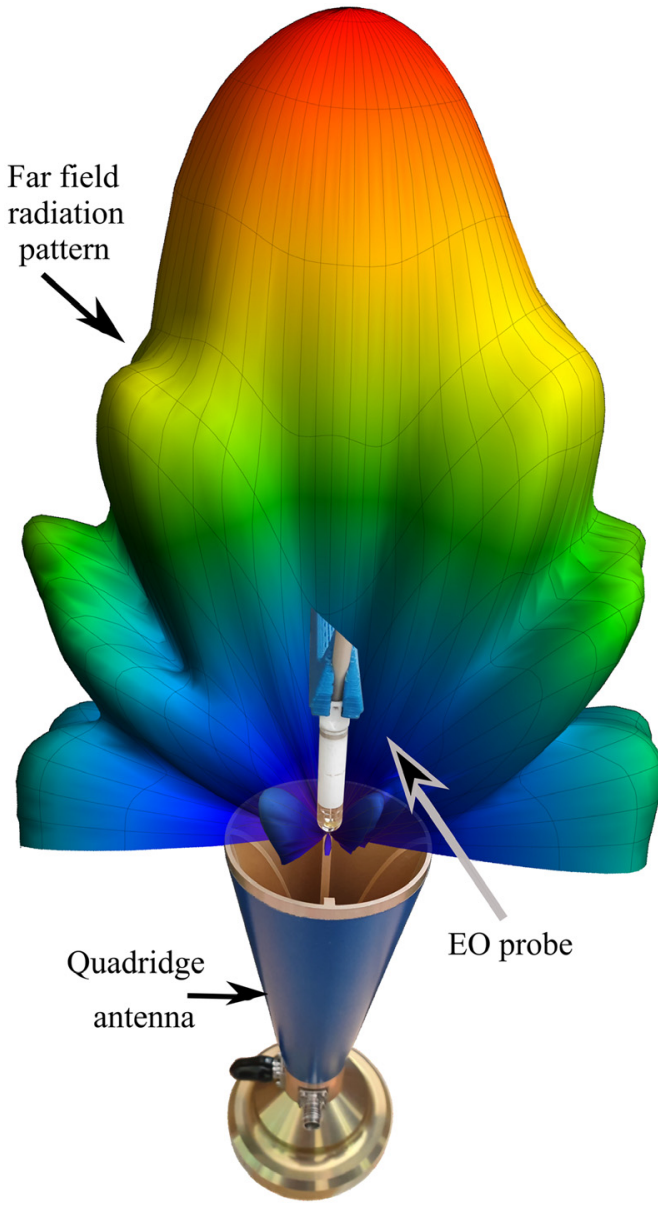

(a)

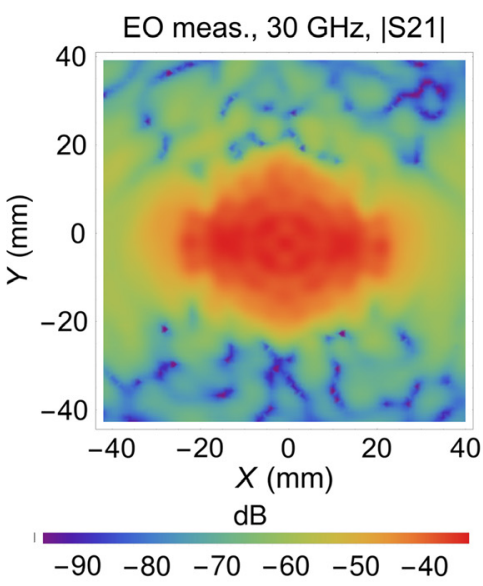

(b)

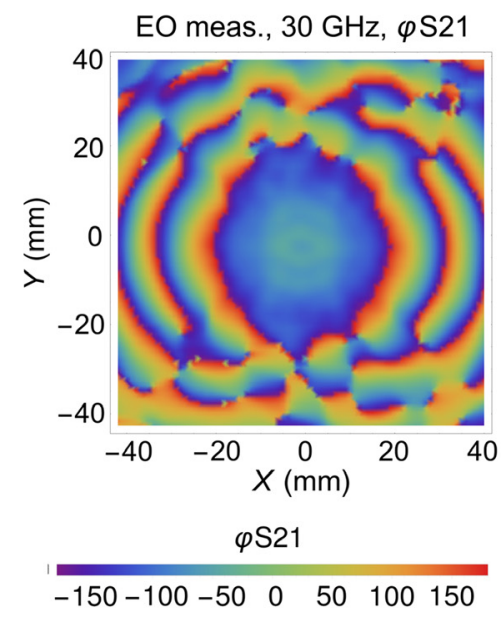

(c)

Fig. 2 (a) Far-field radiation pattern of a closed boundary quadridge antenna (QR18000 from MVG) deduced from the near-field pattern. The EO probe is used to perform the near field analysis in a plane at $2 \mathrm{~mm}$ from the antenna aperture. (b), (c) Near-field distribution in magnitude and phase of the main polarization, measured with the EO probe. Each pixel of the vectorial mapping is considered as secondary spherical source to calculate the far-field pattern. 
Table 1 Comparison of optical sensors. The BW, sensitivity, maximal E-field, footprint, and volume resolution are given for different configurations (config.) and materials. CPI, common path interferometer; MZI, Mach-Zehnder interferometer, seg., segmented electrodes, TFLN, thin film $\mathrm{LiNbO}_{3}$; and $\mathrm{PhC}$, photonic crystal. The footprint corresponds to width $\times$ thickness $\times$ length. The volume resolution corresponds to the square of the lateral resolution times the longitudinal resolution. The lateral resolution is deduced from the optical beam cross section inside the EO crystal, and the longitudinal resolution is estimated from the active length.

\begin{tabular}{|c|c|c|c|c|c|c|}
\hline Reference & Config., material & BW & $\begin{array}{c}\text { Sensitivity } \\
\left(\mathrm{V} \mathrm{m}^{-1} \mathrm{~Hz}^{-1 / 2}\right)\end{array}$ & $\begin{array}{c}E_{\max } \\
(\mathrm{kV} / \mathrm{m})\end{array}$ & Footprint $\left(\mathrm{mm}^{3}\right)$ & $\begin{array}{l}\text { Volume } \\
\text { resolution } \\
\left(\mathrm{mm}^{3}\right)\end{array}$ \\
\hline 9 and 10 & Modulated laser & $500 \mathrm{kHz}$ to $3 \mathrm{GHz}$ & $10 \times 10^{-6}$ & $10^{3}$ & $6.6 \times 6.6 \times 42$ & - \\
\hline 50 & PSM and BSO & $30 \mathrm{~Hz}$ to $60 \mathrm{GHz}$ & $40 \times 10^{-3}$ & $10^{4}$ & $\pi \times(5.5 / 2)^{2} \times 1$ & $\approx(0.1)^{2} \times 1$ \\
\hline 54 & $\mathrm{CPI}$ and $\mathrm{LiNbO}_{3}$ & $20 \mathrm{~Hz}$ to $10 \mathrm{MHz}$ & - & 27 & $40 \times 11.3 \times 180$ & $\approx(0.01)^{2} \times 18$ \\
\hline 55 & $\mathrm{MZI}$ and $\mathrm{LiNbO}_{3}$ & $\mathrm{DC}$ to $20 \mathrm{GHz}$ & $0.1 \times 10^{-3}$ & 10 & $11 \times 8 \times 50$ & $\approx(0.01)^{2} \times 30$ \\
\hline 56 & $\begin{array}{l}\text { Half-MZI seg. } \\
\text { and } \mathrm{LiNbO}_{3}\end{array}$ & $10 \mathrm{kHz}$ to $20 \mathrm{GHz}$ & $10 \times 10^{-3}$ & 0.7 & $10 \times 5 \times 35$ & $\approx(0.01)^{2} \times 12$ \\
\hline 57 & $\mu$ ring and TFLN & up to $100 \mathrm{GHz}$ & $500 \times 10^{-3}$ & - & $5 \times 0.5 \times 15$ & $\begin{array}{c}\approx 7 \times 10^{-4} \times \\
(0.1) 5^{2}\end{array}$ \\
\hline 58 & $\mathrm{PhC}$ and $\mathrm{LiNbO}_{3}$ & $\begin{array}{l}\text { DC to } 5.9 \mathrm{THz} \\
\text { (calculated) }\end{array}$ & 32 & - & $\begin{array}{l}(0.05)^{2} \times \\
7 \times 10^{-4}\end{array}$ & $\begin{array}{c}(0.014)^{2} \times \\
7 \times 10^{-4}\end{array}$ \\
\hline
\end{tabular}

sensitivity, and feasibility. If combined in a two-port pigtailed system, the probe allows measuring simultaneously two orthogonal components of the ambient $E$-field, with a temperatureindependent response, in real time. ${ }^{59}$

Nonetheless, the PSM architecture made of bulky assembled optical elements stands also as an inconvenience for the probes' robustness. Fabry-Perot-based sensors allow this issue to be overcome by changing the polarization interferences into interferences between successive beams reflected at the crystal's facets, as schematically depicted in Fig. 3(a). This approach releases the need for polarizers while also enhancing both sensitivity and longitudinal resolution. ${ }^{36,60-62}$ As shown in Fig. 3(b), the operating point corresponds to the maximal slope of the optical response, at $25 \%$ of the maximal reflection. ${ }^{62}$ Hence, the sensitivity depends on the quality factor, itself linked to the reflective coating. ${ }^{61,62}$ With the quality factor of $Q=1.1 \times 10^{5}$ demonstrated by Lee et al. ${ }^{61}$ for a $350-\mu \mathrm{m}$-long $\mathrm{LiNbO}_{3}$-based FP, a shot-noise-limited sensitivity of $1.2 \mathrm{mV} \mathrm{m}^{-1} \mathrm{~Hz}^{-1 / 2}$ is expected, as estimated from Ref. 62. By orienting light propagation along the crystal's optical axis, the EO response becomes polarization-independent, enhancing the sensor stability toward fiber bending. ${ }^{63} \mathrm{~A}$ two probe-calibration steps associated with a photonic heterodyne scheme was proposed in 2016 by Lee et al. to take benefit from two

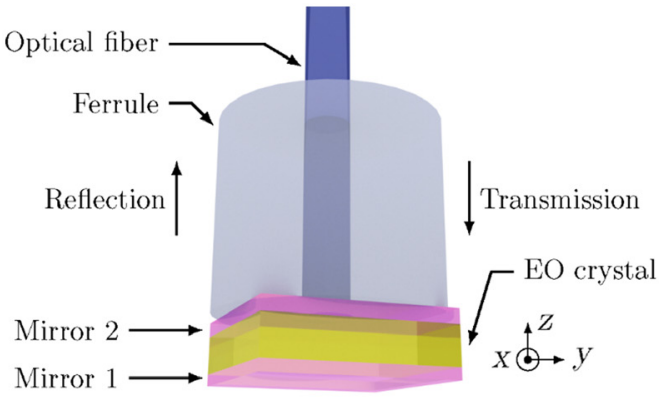

(a)

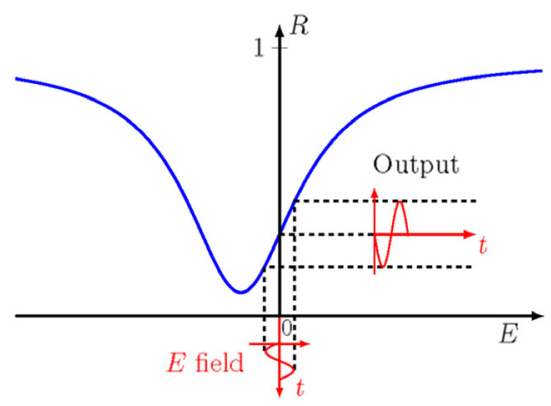

(b)

Fig. 3 Basic configuration of a Fabry-Perot-based EO sensor: (a) schematic view of the configuration and (b) EO response of the sensor. 
probes ensuring respectively minimised invasiveness ${ }^{36}$ and optimized sensitivity, which allowed the characterization of a W-band gyrotron at $95 \mathrm{GHz} .{ }^{37}$ However, the FP response is strongly linked to the facets quality and alignment ${ }^{30}$ and the phase dependency toward temperature also contributes to the operating point instability, which hampers the production reproducibility of FP-based sensors.

In 2012, Hisatake et al. proposed another non-polarimetric approach, based on measuring the $E$-field directly from the phase-modulation sidebands. ${ }^{64}$ In Ref. 65 , the sideband detection by self-heterodyning led to the mapping of near-fields and propagating continuous $\mathrm{THz}$ fields $(125 \mathrm{GHz}$ ), with an SNR of $27 \mathrm{~dB}$. In 2017, the same team added a second EO probe as a reference for phase cancellation. ${ }^{43}$ However, the method remains limited to sub-GHz BW around the central frequency. ${ }^{66}$

In summary, bulk EO sensors are particularly attractive for $E$-field measurements in harsh environments, as well as in the near vicinity of antennas. Their operability at several $\mathrm{THz}$ and their resistance to high fields up to MV/m are two strong characteristics of this type of probe. Yet, bulk probes require a compromise between sensitivity, stability, and lateral resolution. Hence, an mm-long EO probe with a GRIN lens shows a 100- $\mu \mathrm{m}$ lateral resolution, as shown in Table 1. Integrated EO sensors overcome this issue by confining light within micrometric cross sections, without any additional focusing element. We propose to classify the integrated $E$-field EO probes into two categories: waveguide-based probes and photonic probes, differentiated by the size of their optical waveguides.

\section{Waveguide-Based Electro-Optic Sensors}

$\mathrm{LiNbO}_{3}$ integrated optics was initiated in the 1970s by Bell Labs ${ }^{67}$ and NTT Corporation, ${ }^{68}$ and it soon opened the way to planar integrated interferometers. ${ }^{69}$ Among them, Mach-Zehnder (MZ) and common path interferometer (CPI) ${ }^{27}$ stand out for their excellent linearity due to their sinusoidal response, similar to PSM-based bulk sensors:

$$
I_{\mathrm{out}}=\frac{I_{S}}{2}\left(1+b \cdot \cos \left(\varphi_{0}+\frac{\pi \cdot E}{E_{\pi}}\right)\right)
$$

where $I_{\text {out }}$ is the output irradiance, $I_{S}$ is a referenced fixed irradiance, $b$ stands for extinction ratio, $E_{\pi}$ is the so-called "half-wave electric field," namely the electric field required to change the output irradiance by half a period, $E$ is the external electric field amplitude, and $\varphi_{0}$ is the phase bias, which is set to $\pi / 2$ to ensure linearity, similarly as in the bulk configuration [see Fig. 1(b)].

When thermal stability is a critical issue, e.g., for voltage measurement or intense $E$-field detection, the CPI configuration is preferred. ${ }^{54,70-72}$ The configuration is shown in Fig. 4(a): it

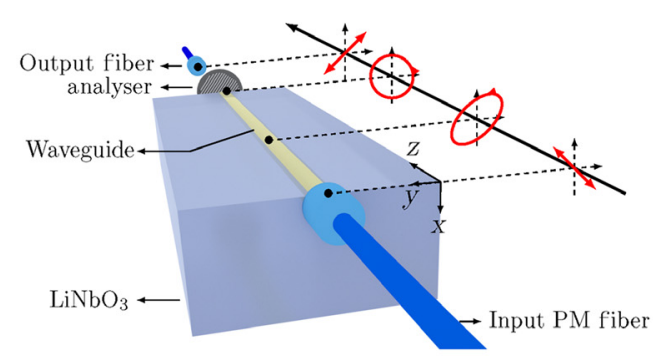

(a)

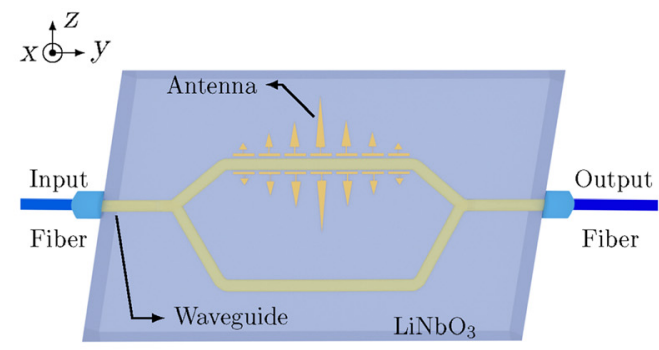

(b)

Fig. 4 Schematic views of integrated EO sensors: (a) CPI. The configuration is the same as in a SPM-based sensor, expect that a waveguide is integrated in the EO crystal, which releases the need of focusing element. With an adjusted length, the waveguide behaves as a quarter-waveplate. (b) Mach-Zenhder interferometer (MZI). The waveguide is usually polarizing (made by proton-exchange), and it is butt-coupled to PM or single-mode (SM) fibers. Antennas are potentially used to locally enhance the electric field. 
relies on a single channel waveguide, usually made by Ti-diffusion. The two interfering beams correspond to the two quasi-TE and quasi-TM propagating polarizations. They are equally excited by an input PM fiber with a neutral axis oriented at 45 deg to the (Ox) crystal axis and recombined at the waveguide output by a polarizer, also oriented at 45 deg to the crystal axis. Overall, this architecture represents the integrated version of the PSM-based sensors.

The phase bias $\varphi_{0}=2 \pi \cdot \Delta n \cdot L / \lambda$ can be fixed easily by adjusting the waveguide length $L$. CPIs are usually oriented along the $(\mathrm{Oz})$ axis, which offers minimal birefringence $\Delta n$ (between $10^{-5}$ and $10^{-4} \mathrm{RIU}^{71}$ ), and thereby also minimal thermal fluctuations of $\varphi_{0}$. To cancel the predominant contribution of the pyroelectric effect, a conductive and transparent layer such as tin oxide ITO can be deposited on both $Z$ waveguides facets and connected together. ${ }^{71}$ Phase bias variations as low as $1.5 \times 10^{-3} \mathrm{~K}^{-1} \mathrm{~mm}^{-1}$ are reported, ${ }^{72}$ which is the best thermal stability reported to date in an integrated $\mathrm{EO} \mathrm{LiNbO}_{3}$ sensor. Increased sensitivity can be achieved by a minimally invasive antenna deposited on both sides of the waveguide,$^{54}$ or by locally thinning the substrate with a dicing saw as shown in Fig. 5. This latter approach, also described in Ref. 73, spurs the EO overlap up to a factor 40 if the electrodes are also structured.

The EO efficiency of CPI-based sensors is governed either by $r_{22} \cdot n_{\mathrm{o}}{ }^{3}$ for Z-propagating guides ${ }^{54}$ or by $\left(r_{33} \cdot n_{\mathrm{e}}{ }^{3}\right.$ to $\left.r_{13} \cdot n_{\mathrm{o}}{ }^{3}\right) / 2$ in the other cases, ${ }^{74,75}$ where $n_{\mathrm{o}}$ and $n_{\mathrm{e}}$ are the extraordinary and ordinary refractive indices. However, $r_{13}$ and $r_{22}$ are more than three times lower than $r_{33}$. As a consequence, CPIs give way to other configurations exploiting $r_{33}$ when sensitivity becomes a critical parameter, e.g., for electromagnetic compatibility or SAR tests.

In MZIs, the output irradiance expressed in Eq. (1) comes from the interference between the two branches seen in Fig. 4(b): light enters the sensor via a single channel and is then split into two arms through a $Y$ junction. The two branches show a different EO sensitivity so that when the two signals recombine through another $Y$ junction into the output waveguide, their phase difference is proportional to the external electric field. ${ }^{69,75-77}$ MZIs were the subject of intensive efforts in the 1980s and 1990s for E-field detection, as detailed in Ref. 27. Now, the usual configuration employs $X$-cut $Y$-propagative waveguides to benefit from the $r_{33}$ coefficient while avoiding the strong pyroelectric effect of the $Z$-cut substrates. The differentiated EO sensitivity between the two arms is achieved either by reverse poling ${ }^{78}$ or by antennas. ${ }^{27,69,75,77,79,80}$ The phase bias $\varphi_{\mathrm{o}}$ is set to $\pi / 2$ by a length difference of several micrometers between the two arms and can be corrected by applying force ${ }^{81}$ or by laser writing ${ }^{79,82}$ in case of fabrication imprecisions.

As the two interfering beams do not follow the same path, MZIs are very sensitive to external fluctuations such as temperature. For example, recent measurements with proton-exchangedbased polarized MZIs show a bias variation of $0.12 \mathrm{~K}^{-1} \mathrm{~mm}^{-1},{ }^{79}$ two orders of magnitude higher than in CPIs. Therefore, efforts since 2012 have mainly focused on bias stabilization.
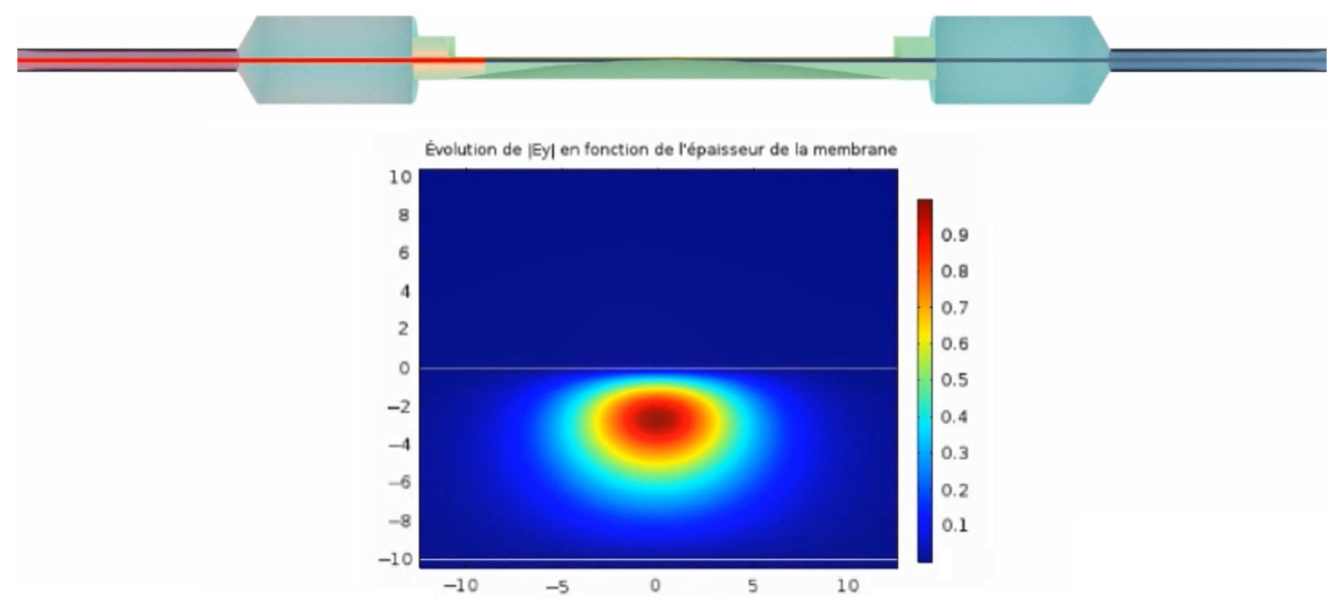

Fig. 5 Fabrication of a locally thinned CPI-based sensor. The input and output fibers are polarized. Locally, the waveguide is thinned down to micrometric or submicrometric thickness by precise dicing. The EO overlap is thereby enhanced by locally confining the light, whereas the IL are kept low. ${ }^{73}$ (Video 1, MP4, 56 MB [URL: https://doi.org/10.1117/1.JOM.1.2.020902.1]). 
Alternatively to wavelength tuning, ${ }^{83}$ a photovoltaic power-over-fiber module was developed by SRICO Corp. to provide remote control of the bias. ${ }^{84}$ Another approach proposed by the same group is to monitor the received optical power using the ratio of harmonics, which is operatingpoint independent. ${ }^{85}$ As an alternative, the INAOE institute adds a second asymmetric MZI optical retarder to adjust the operating point. ${ }^{86}$ Thus various solutions are now available for bias control but requiring an additional control system.

The most attractive features of $\mathrm{LiNbO}_{3}$ MZIs is their sensitivity, which adds to the use of $r_{33}$ the benefit of low propagation losses $(<0.1 \mathrm{~dB} / \mathrm{cm}):{ }^{87}$ a centimetric active length has thereby no impact on the insertion losses (IL), which is of crucial importance for improving the sensitivity. ${ }^{88}$ As an example, the SRICO MZI modulators show a sensitivity of $100 \mu \mathrm{V} \mathrm{m}^{-1} \mathrm{~Hz}^{-1 / 2}$ due to a total length of $5 \mathrm{~cm}$ with IL $<4.0 \mathrm{~dB}$ (see Table 1). Coplanar antennas also play a prominent role in MZIs sensitivity. ${ }^{27}$ Among the numerous designs, segmented antennas ${ }^{80}$ distinguish themselves by their ability to provide impedance matching and wide BW. For example, Kumming University has recently proposed a segmented tapered dipole antenna to achieve large BW without compromise on sensitivity, where gradual changes in antennas size allow covering a $10-\mathrm{kHz}$ to $20-\mathrm{GHz}$ BW with a sensitivity of $10 \mathrm{mV} \mathrm{m}^{-1} \mathrm{~Hz}^{-1 / 2}$ and a total length of $3.5 \mathrm{~cm}^{56}$ (see Table 1). Noteworthy, replacing the broadband antenna with resonant ones also opens the way to advanced radio over fiber systems for wireless telecommunications. ${ }^{89}$ Despite their metallic parts, MZIs with antenna can be used in harsh environments if properly encapsulated, as demonstrated by Seikoh-Giken Corp. ${ }^{24}$

However, the centimeter footprint of the MZI is a hindrance to the minimally invasive measurement of $E$-fields. Reflection-based configurations ${ }^{24,79}$ allow to address this issue and ease manipulation: the $\mathrm{MZ}$ is half-cut and ends with a mirror so that only one fiber is required. But the back light fluctuations result then in degraded sensitivity. ${ }^{79}$

Since 2010, the commercial introduction of $\mathrm{LiNbO}_{3}$ thin films (TFLN) ${ }^{57,90,91}$ based on ion slicing technology ${ }^{92}$ has allowed a decisive step toward miniaturization. By confining light in sub- $\mu \mathrm{m}$-wide waveguides, TFLNs open the way to low-loss micrometer-scale curvature radius, which drastically reduces the MZIs dimensions. They also help to diminish the gap between antennas, which enhances the local $E$-field and decreases the active length, releasing thereby the BW. Hence, LNTF-based MZ modulators are now available with BWs exceeding $60 \mathrm{GHz}$ and a millimeter footprint ${ }^{57,93,94}$ and should give rise to EO $E$-field probes with similar performances. The still mitigated sensitivity $\left(500 \mathrm{mV} \mathrm{m}^{-1} \mathrm{~Hz}^{-1 / 2}\right.$, see Table 1 ) should evolve with the improvement of the IL, which are now of $8 \mathrm{~dB} .^{57,93}$

In summary, lithium niobate appears to be a material of choice for the production of waveguide-based probes due to its attractive EO coefficients and technological manufacturing maturity. The revolution initiated in $\mathrm{LN}$ photonic integrated circuits thanks to ion slicing offers the opportunity to reduce the size of $\mathrm{LiNbO}_{3}$ components down to millimeter sizes. This revolution associated with the deployment of specific technologies such as epitaxy or precise dicing also open up perspectives on varied materials, such as $\mathrm{GaP}^{95}$ which allows large $\mathrm{BW}$, $\mathrm{BSO}$, which provides excellent thermal stability, ${ }^{96}$ or $\mathrm{BaTiO}_{3}$, which ensures exceptional EO efficiency. ${ }^{97}$

The growing demand for minimized disturbance, high portability, and ultra-large BW pushes now toward further miniaturization. It stimulates new photonic architectures with overall micrometric or submicrometric size.

\section{Photonic EO Probes}

The quest for ultra-compact EO probes requires compensating for the minimized active lengths by architecture exalting sensitivity, whether through resonance, extreme EM confinement, or slow light effects.

In the 2000s, resonant fibered structures with submillimetric sizes, such as EO racetrack resonators ${ }^{98}$ or slabs, ${ }^{99}$ were directly coupled to the side of polished fibers to form slab-coupled optical sensors, also opening the way to multi-axis sensing. ${ }^{100}$ However, a cm-long encapsulation was necessary along the fiber to avoid bending in the detection zone.

By bonding submicrometer thin films of $\mathrm{LiNbO}_{3}$ to silicon microring resonators, the Ohio State University demonstrated a more robust and compact approach, combining the advantage of 
silicon integrated optics in terms of cost and density of integration with the attracting EO properties of $\mathrm{LiNbO}_{3} \cdot{ }^{101}$ With a silicon microring radius as small as $20 \mu \mathrm{m}$ and a quality factor of $Q=13,000$, they achieved a probe with a sensitivity of $4.5 \mathrm{~V} \mathrm{~m}^{-1} \mathrm{~Hz}^{-1 / 2}$. Thus the longitudinal resolution gains two orders of magnitude compared to CPIs or MZIs while ensuring also an attractive sensitivity. And a further two-orders-of-magnitude improvement in the sensitivity is expected for $Q$ exceeding $10^{6}$, which is now achievable. ${ }^{102,103}$ However, a giant $Q$ also decreases the $\mathrm{BW}$ due to the increased resonator lifetime: $\mathrm{BW}=c /(\lambda \cdot Q)$, where $c$ is the light velocity in vacuum. Thus probes with an above-million quality factor cannot exceed a $\mathrm{GHz} \mathrm{BW}$.

An alternative to resonators relies on exploiting ultra-high confinement of the electric field in the sensing region through plasmonic architectures. If combined with polymers showing high EO efficiencies and THz BW (see, for example, Refs. 104 and 105), this approach promises to meet the challenge of both large BW and enhanced sensitivities. Hence, the ETH Institute and Washington University proposed a bowtie antenna to play the role of a plasmonic phase modulator as well as of an $E$-field enhancer. ${ }^{44}$ The $2.2 \times 5 \mu \mathrm{m}^{2}$ gap of the bowtie antenna was filled with EO JRD1 in polymethyl methacrylate polymer, showing an EO efficiency of $170 \mathrm{pm} / \mathrm{V}$, five times higher than in lithium niobate. Thereby, an interactive length of only $4 \mu \mathrm{m}$ sufficed to detect electric fields lower than $10 \mathrm{~V} / \mathrm{m}$ over a $1.25-\mathrm{THz} \mathrm{BW}$. Afterward, to replace this freespace setup with an on-chip detection system with a higher EO overlap, the same team proposed a silicon-based MZI with two antenna-coupled plasmonic phase shifters on each branch, the antenna being either a bowtie, ${ }^{106}$ or a multi-resonant antenna ${ }^{107}$ as represented in Fig. 6.

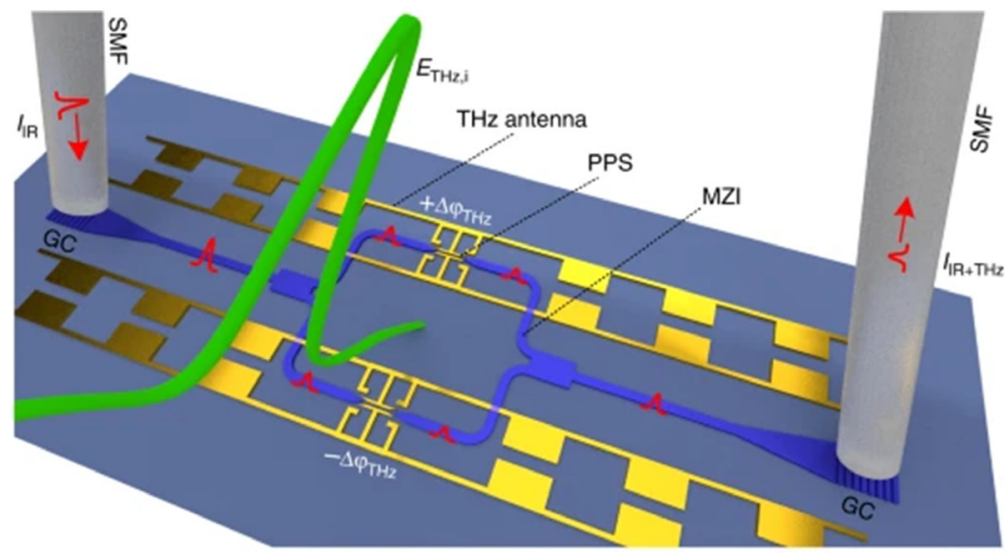

(a)

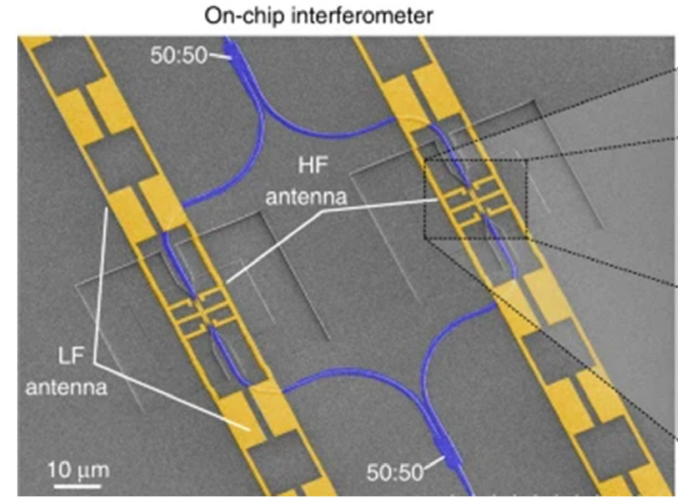

(b)

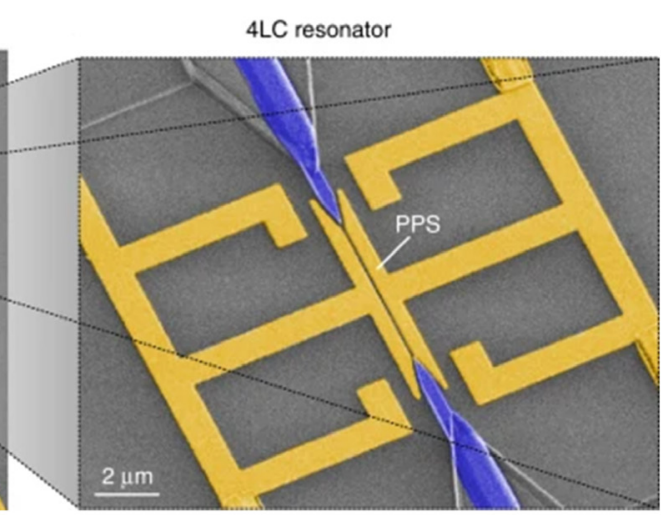

(c)

Fig. 6 On-chip terahertz detector. ${ }^{107}$ (a) The light pulses are coupled in and out on-chip silicon (Si) waveguides by means of grating couplers. An organic EO material fills the two-phase shifters with opposite polarity to enable push-pull operation. Scale bar is $10 \mu \mathrm{m}$. (b) False color scanning electron image of the fabricated multi-resonant $\mathrm{THz}$ detector. The antenna comprises of a highfrequency (HF) antenna and a low-frequency (LF) antenna. (c) Close-up view of the HF-THz antenna. Reproduced with permission from Refs. 107 and 108. 
Compared to bulk, the multi-resonant antenna provides a three-orders-of-magnitude enhancement of the THz-fields with $2.5 \mathrm{THz}$ BW. Hence, the 5- $\mu$ m-long plasmonic zones show an EO efficiency 35 times higher than a 1-mm-long bulk ZnTe crystal at $2.4 \mathrm{THz} \cdot{ }^{107}$ However, the in/ output couplers are still non-adapted industrial applications, as can be judged by the overall $-31 \mathrm{~dB}$ losses.

Alternatively to plasmonic structures, photonic crystals (PhCs) can enhance EO interaction by slow-light effects. ${ }^{109,110}$ In other words, the small active length is compensated by an increased interaction time due to the excitation of a small group velocity Bloch mode. The gain in EO efficiency as compared to a bulk crystal is proportional to $f^{2}$, where $f$ is the local field factor of the optical wave: ${ }^{10,111}$

$$
f=\sqrt{\frac{v_{g, \text { bulk }}}{v_{g, \mathrm{PhC}}}}
$$

where $v_{g \text {,bulk }}$ and $v_{g \text {,PhC }}$ denoting the group velocities in the bulk EO material and in the $\mathrm{PhC}$, respectively. ${ }^{112}$ Thus in 2014 , the Texas University showed that a $300-\mu \mathrm{m}$-long slot PhCwaveguide filled with SE125 EO polymer $(r 33=125 \mathrm{pm} / \mathrm{V})$ and surrounded with a bowtie antenna was able to detect electric fields as small as $2.5 \mathrm{~V} / \mathrm{m}$ at $8.4 \mathrm{GHz}{ }^{45}$ It represented a gain of 14 in EO efficiency compared to the bulk EO polymer. However, fiber coupling was achieved with gratings, which both hampered the robustness and spurred the optical losses.

A simple solution to integrate the $\mathrm{PhC}$ is to place it directly at the fiber output, as represented in Fig. 7. Hence, Calero et al. ${ }^{58}$ developed an all-dielectric ultra-compact fibered EO sensor with an active length of only $700 \mathrm{~nm}$ and a sensing area of $14 \times 14 \mu \mathrm{m}^{2}$, pushing the limits toward EM invisibility.

The PhC-based sensor consists of an $X$-cut lithium niobate thin film, designed to exhibit a Fano-resonance at $1575 \mathrm{~nm}$ thanks to an optimized biperiodic square lattice pattern. ${ }^{113}$ The Fano-response of the reflected spectrum characterizes itself by an asymmetric resonance [see Fig. 8(a), black curve], with a sharp spectral slope promoting slow light and high sensitivity toward $E$-field induced refractive index variations. The agreement between the slope calculation (in red) and the measured EO strength (in blue) confirms the contribution of the Fano resonance to the EO modulation. The extreme miniaturization combined with the absence of electrodes benefits the BW, which theoretically extends up to $5.9 \mathrm{THz}$. In addition, the lateral resolution is improved by one order of magnitude compared to the bulky EO sensors [see Fig. 8(b)].

The performances of the sensor are summarized in Table 1 and compared with the other configurations. The still weak sensitivity of $32 \mathrm{~V} \mathrm{~m}^{-1} \mathrm{~Hz}^{-1 / 2}$ will advantageously be improved

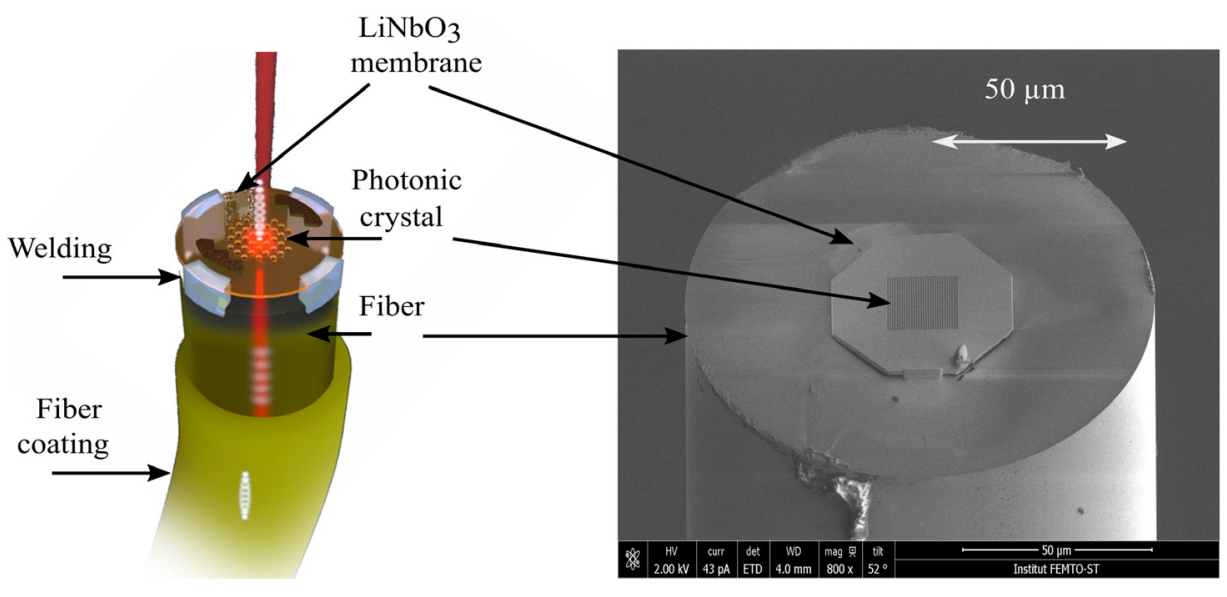

(a)

(b)

Fig. 7 Basic principle of PhC-based EO sensor: (a) sketch of the sensor showing the assembly between the PhC and the PM fiber and (b) SEM view of the PhC-based sensor. 


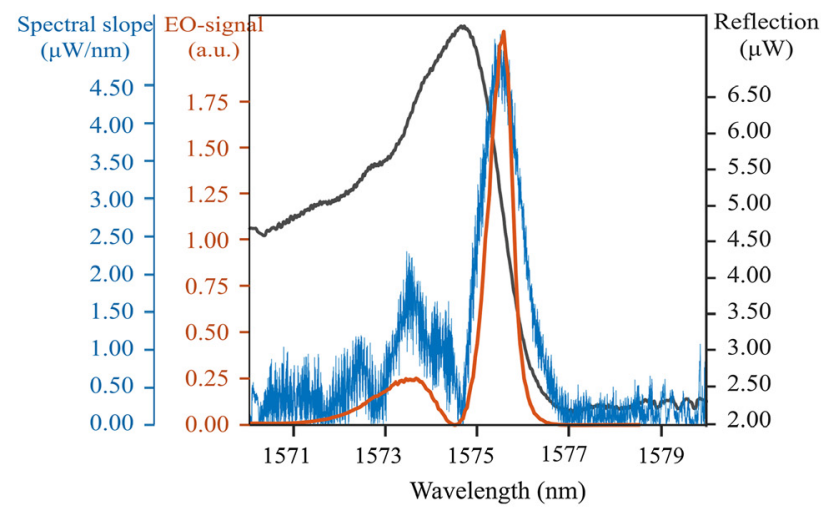

(a)

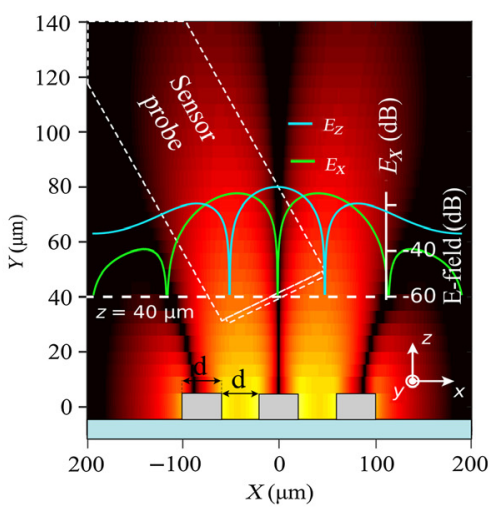

(b)

Fig. 8 Performances of the $\mathrm{LiNbO}_{3} \mathrm{PhC}$-based sensor. (a) Theoretical reflected spectrum of the Fano resonance (black) represented with his slope value (blue) and the EO-modulation strength in function of the laser source wavelength (red). (b) Finite-element-method-computed electric field distribution produced by the coplanar lines. The green and blue curves represent, respectively, the $E_{x}$ and $E_{z}$ components along the $x$ axis at a distance of 40 away from the lines. The associated experimental results are developed in Ref. 58 and show excellent agreement with computation.

in the future by adding an antenna, reducing the laser back reflection, or exploiting dark Fano resonances. $^{114}$

Overall, photonic probes appear as excellent candidates to move toward minimal invasiveness and THz frequency BW. Despite the still possible improvement in sensitivity, they are nonetheless already attractive for application in high fields such as cold plasmas.

\section{Conclusion}

In summary, compared to their active optical counterparts (row \#1, Table 1), EO probes show the ability to cover wider BWs with lower induced perturbation. The EO probes differ from each other by the nature of their material and their photonic architecture: their specificities are summarized in Table 1. In bulk sensors, numerous varieties of materials are used, from cubic ferroelectric materials privileging temperature-stability to polymers or anisotropic EO crystals for sensitive applications. With their all-dielectric feature and their typical millimetric footprint, bulk EO probes show a sensitivity of tens of $\mathrm{mV} \mathrm{m}^{-1} \mathrm{~Hz}^{-1 / 2}$ and a $\mathrm{BW}$ covering more than 10 frequency decades. Due to their excellent technological maturity, their developments over the past 10 years have been mostly dedicated to specific applications like radiation pattern imaging or for measuring $E$-fields in cold plasmas, RMI devices, or RF devices. In comparison, integrated EO probes allow mono-block architectures with a smaller lateral resolution, down to the micrometer. The material of choice is lithium niobate, with two privileged architectures: CPI-based sensors for $T$-stabilized evaluations and MZIs for sensitive measurements down to $100 \mu \mathrm{V} \mathrm{m}^{-1} \mathrm{~Hz}^{-1 / 2}$. They also benefit from technological maturity and are used in harsh environments as well. The recent development of $\mathrm{LiNbO}_{3}$ thin films opens the way to new integrated devices with smaller waveguides allowing gain both in spatial resolution and BW. Finally, a new generation of photonic EO probes is appearing, revealing other attractive materials such as polymers with enhanced EO coefficients. Photonic EO probes show the advantage of unprecedented spatial longitudinal resolution, down to $700 \mathrm{~nm}$ for PhCs. Their ultra-small footprint allows the micrometric localization of hot spots and tends to EM invisibility.

These developments pave the way to dense arrays of 2D EO probes for real-time imaging, pushing the limits one or two orders of magnitudes beyond the current set of 2 or 3 probes dedicated to vector or isotropic measurements. ${ }^{115}$ The now-possible detection of faint terahertz $E$-fields also contributes to new applications such as ultra-fast nanoscopy or quantum electrodynamics. ${ }^{116}$ The future tends to combine these EO heads with numerical approaches such as deep learning to facilitate diagnosis or localization, ${ }^{117}$ or such as imaging reconstruction algorithms combined with metasurfaces ${ }^{118}$ to allow single shot single sensor imaging. 


\section{Acknowledgments}

A part of this work has received funding from the French National Research Agency (ANRAgence Nationale pour la Recherche) under the Grant Agreement Nos. CEPAGE ANR-16CE24-0024-01 and 19 LCV2 0007 01-SYRAH-lab. It was also partially supported by the I-SITE BFC project (Contract No. ANR-15-IDEX-0003). The technological developments performed at FEMTO-ST were developed within the Renatech network facilities. The authors would like to thank Anthony Perignon and Chloé Courjal for the video mounting.

\section{References}

1. ICNIRP, "Guidelines for limiting exposure to electromagnetic fields (100 kHz to 300 GHz)," Health Phys. 118(5), 483-524 (2020).

2. A. Hirata, D. Funahashi, and S. Kodera, "Setting exposure guidelines and product safety standards for radio-frequency exposure at frequencies above $6 \mathrm{GHz}$ : brief review," Ann. Telecommun. 74, 17-24 (2019).

3. E. Chiaramello et al., "Radio frequency electromagnetic fields exposure assessment in indoor environments: a review," Int. J. Environ. Res. Public Health 16, 955 (2019).

4. "Electromagnetic compatibility (EMC) Part 4-22: testing and measurement techniquesradiated emission and immunity measurements in fully anechoic rooms (FARs)," CEI EN 61000-4-22 (2014).

5. H. Wang et al., "The specific absorption rate in different brain regions of rats exposed to electromagnetic plane waves," Sci. Rep. 9, 13277 (2019).

6. Z. Vilagosh, A. Lajevardipour, and A. Wood, "Computer simulation study of the penetration of pulsed 30, 60, and $90 \mathrm{GHz}$ radiation into the human ear," Sci. Rep. 10, 1479 (2020).

7. https://www.dare.eu/electric-field-probes/radisense-10.

8. N. M. Burford and M. O. El-Shenawee, "Review of terahertz photoconductive antenna technology," Opt. Eng. 56(1), 010901 (2017).

9. L. Winter et al., "Parallel transmission medical implant safety testbed: real-time mitigation of RF induced tip heating using time-domain E-field sensors," Magn. Reson. Med. 84, 3468-3484 (2020).

10. http://www.enprobe.de/products_FO-Antennas.htm.

11. R. D. Mehlenbacher et al., "Nanomaterials for in vivo imaging of mechanical forces and electrical fields," Nat. Mater. 3, 17080 (2018).

12. A. Habib et al., "Electro-plasmonic antenna: a nonfluorescent optical probe for ultrasensitive label-free detection of electrophysiological signals," Sci. Adv. 5(10), eaav9786 (2019).

13. A. L. Efros et al., "Evaluating the potential of using quantum dots for monitoring electrical signals in neurons," Nat. Nanotechnol. 13, 278-288 (2018).

14. J. Sedlacek et al., "Atom-based vector microwave electrometry using rubidium Rydberg atoms in a vapor cell," Phys. Rev. Lett. 111, 063001 (2013).

15. S. Kumar et al., "Atom-based sensing of weak radio frequency electric fields using homodyne readout," Sci. Rep. 7, 42981 (2017).

16. H. Fan et al., "Atom based RF electric field sensing," J. Phys. B Mol. Opt. Phys. 48(20), 202001 (2015).

17. M. Jing et al., "Atomic superheterodyne receiver based on microwave-dressed Rydberg spectroscopy," Nat. Phys. 16, 911-915 (2020).

18. C. L. Holloway et al., "Broadband Rydberg atom-based electric-field probe for SI-traceable, self-calibrated measurements," IEEE Trans. Antennas Propag. 62(12), 6169-6182 (2014).

19. S. Namba, "High voltage measurement by ADP crystal plate," Rev. Sci. Instrum. 32, 595 (1961).

20. G. A. Massey, D. C. Erickson, and R. A. Kadlec, "Electromagnetic field components: their measurement using linear electrooptic and magnetooptic effects," Appl. Opt. 14(11), 2712-2719 (1975).

21. Q. Wu and X.-C. Zhang, "7 terahertz broadband GaP electro-optic sensor," Appl. Phys. Lett. 70, 1784 (1997). 
22. S. Keiber et al., "Electro-optic sampling of near-infrared waveforms," Nat. Photonics 10, 159-162 (2016).

23. P. Sulzer et al., "Determination of the electric field and its Hilbert transform in femtosecond electro-optic sampling," Phys. Rev. A 101, 033821 (2020).

24. M. Nishiura et al., "Electro-optic probe measurements of electric fields in plasmas," Rev. Sci. Instrum. 88, 023501 (2017).

25. F. Consoli et al., "Time-resolved absolute measurements by electro-optic effect of giant electromagnetic pulses due to laser-plasma interaction in nanosecond regime," Sci. Rep. 6, 27889 (2016).

26. W. J. Koshak and R. J. Solakiewicz, "Electro-optic lightning detector," Appl. Opt. 38(21), 4623 (1999).

27. R. Zeng et al., "Development and application of integrated optical sensors for intense E-field measurement," Sensors 12(8), 11406-11434 (2012).

28. H. Togo, S. Mochizuki, and N. Kukutsu, "Optical fiber electric field sensor for antenna measurement," NTT Tech. Rev. 7(3), 1-6 (2009).

29. D.-J. Lee et al., "Recent advances in the design of electro-optic sensors for minimally destructive microwave field probing," Sensors 11, 806-824 (2011).

30. L. Duvillaret, S. Rialland, and J.-L. Coutaz, "Electro-optic sensors for electric field measurements. I. Theoretical comparison among different modulation techniques," J. Opt. Soc. Am. B 19, 2692-2703 (2002).

31. F. Blanchard and K. Tanaka, "Improve time and space resolution in electro-optic sampling for near field terahertz imaging," Opt. Lett. 41(20) 4645 (2016).

32. E. Slikboer et al., "Electric field and temperature in a target induced by plasma jet imaged using Mueller polarimetry," J. Phys. D Appl. Phys. 51(2), 025204 (2018).

33. E. Slikboer et al., "Revealing plasma-surface interaction at atmospheric pressure: imaging of electric field and temperature inside the targeted material," Sci. Rep. 10, 2712 (2020).

34. K. Idaka, "Electric field and voltage measurement by using electro-optic sensor," in 11th Int. Symp. High-Voltage Eng. (1999).

35. H. Togo et al., "Characterization of millimeter-wave antenna using photonic measurement techniques," Int. J. RF Microwave Comput.-Aided Eng. 14(3), 290-297 (2004).

36. D.-J. Lee, J.-Y. Kwon, and N.-W. Kang, "Field analyzing of electro-optic probes for minimally invasive microwave sampling," Opt. Express 22(3), 2897-2909 (2014).

37. S. Kim et al., "Two-tier calibrated electro-optic sensing system for intense field characterization of high-power W-band gyrotron," Opt. Express 24, 10547-10555 (2016).

38. K. Sarabandi et al., "Pattern and gain characterization using nonintrusive very-near-field electro-optical measurements over arbitrary closed surfaces," IEEE Trans. Antennas Propag. 65(2), 489 (2017).

39. H. Togo et al., "Sensitivity-stabilized fiber-mounted electrooptic probe for electric field mapping," J. Lightwave Technol. 26(15), 2700-2705 (2008).

40. M. Bernier et al., "Electric field and temperature measurement using ultra wide bandwidth pigtailed electro-optic probes," Appl. Opt. 47, 2470-2476 (2008).

41. L. Duvillaret, S. Rialland, and J.-L. Coutaz, "Electro-optic sensors for electric field measurements. II. Choice of the crystals and complete optimization of their orientation," J. Opt. Soc. Am. B 19, 2704-2715 (2002).

42. B. Pradarutti et al., "Highly efficient terahertz electro-optic sampling by material optimization at 1060 nm," Opt. Commun. 281(19), 5031-5035 (2008).

43. S. Hisatake et al., "Mapping of electromagnetic waves generated by free-running selfoscillating devices," Sci. Rep. 7, 9203 (2017).

44. I.-C. Benea-Chelmus et al., "Three-dimensional phase modulator at telecom wavelength acting as a THz detector with an electro-optic bandwidth of $1.25 \mathrm{THz}$," ACS Photonics 5(4), 1398-1403 (2018).

45. X. Zhang et al., "Integrated photonic electromagnetic field sensor based on broadband bowtie antenna coupled silicon organic hybrid modulator," J. Lightwave Technol. 32(20), 3774-3784 (2014).

46. V. C. McLaughlin et al., "Wideband $15 \mathrm{THz}$ response using organic electro-optic polymer emitter-sensor pairs at telecommunication wavelengths," Appl. Phys. Lett. 92, 151107 (2008). 
47. N. Ticaud et al., "Specific absorption rate assessment using simultaneous electric field and temperature measurements," IEEE Antennas Wireless Propag. Lett. 11, 252-255 (2012).

48. G. Gaborit et al., " $20 \mathrm{GHz}$ antenna radiation pattern obtained from near-field mapping with electrooptic probe on a single plane," IEEE Antennas Wireless Propag. Lett. 19(7), 1177-1181 (2020).

49. E. Roussel et al., "Phase diversity electro-optic sampling: a new approach to single-shot terahertz waveform recording," https://ui.adsabs.harvard.edu/abs/2020arXiv200203782R (2020).

50. https://www.kapteos.com.

51. https://emagtech.com/neoscan-system/.

52. S. Reiss, A. Bitzer, and M. Bock, "An optical setup for electric field measurements in MRI with high spatial resolution," Phys. Med. Biol. 60, 4355-4370 (2015).

53. I. Saniour et al., "Electro-optic probe for real-time assessments of RF electric field produced in an MRI scanner: feasibility tests at 3 and 4.7 T.," NMR Biomed. 31, e3849 (2018).

54. Z. Li et al., "Measurement of distorted power-frequency electric field with integrated optical sensor," IEEE Trans. Inst. Meas. 68(4), 1132-1139 (2019).

55. https://www.srico.com/products/.

56. J. Zhang et al., "Broad-band integrated optical electric field sensor using reflection MachZehnder waveguide modulator," Fiber Integrated Opt. 36(4-5), 157-164 (2017).

57. http://www.partow-tech.com/.

58. V. Calero et al., "An ultra wideband-high spatial resolution-compact electric field sensor based on Lab-on-Fiber technology," Sci. Rep. 9, 8058 (2019).

59. Y. Gaeremynck et al., "Two electric-field components measurement using a 2-port pigtailed electro-optic sensor," Appl. Phys. Lett. 99, 141102 (2011).

60. D. J. Lee and J. F. Whitaker, "A simplified Fabry-Perot electro-optic modulator sensor," IEEE Photonics Technol. Lett. 20, 866-868 (2008).

61. W.-B. Lee, S.-U. Baek, and S.-S. Lee, "Highly sensitive electro-optic probe incorporating an ultra-high $Q$-factor $\mathrm{LiNbO}_{3}$ etalon," Appl. Opt. 57(28), 8343-8349 (2018).

62. G. Gaborit et al., "High-finesse Fabry-Perot electro-optic sensors with enhanced sensitivity and spatial resolution," Appl. Opt. 46, 2001-2009 (2007).

63. S. Kim, Y.-P. Hong, and D. J. Lee, "Polarization insensitive electro-optic probe using birefringence-free interferometric modulation," Opt. Lett. 44(11), 2895-2898 (2019).

64. S. Hisatake and T. Nagatsuma, "Nonpolarimetric technique for homodyne-type electrooptic field detection," Appl. Phys. Express 5, 012701 (2012).

65. S. Hisatake, H. H. N. Pham, and T. Nagatsuma, "Visualization of the spatial-temporal evolution of continuous electromagnetic waves in the terahertz range based on photonics technology," Optica 1(6), 365-369 (2014).

66. S. Hisatake et al., "Visualization of frequency-modulated electric field based on photonic frequency tracking in asynchronous electro-optic measurement system," Appl. Phys. Express 11(4), 046601 (2018).

67. I. P. Kaminow and V. Ramaswamy, "Lithium niobate ridge waveguide modulator," Appl. Phys. Lett. 24, 622 (1974).

68. Y. Ohmachi and J. Noda, "Electro-optic light modulator with branched ridge waveguide," Appl. Phys. Lett. 27, 544 (1975).

69. C. H. Bulmer, W. K. Burns, and R. P. Moeller, "Linear interferometric waveguide modulator for electromagnetic-field detection," Opt. Lett. 5(5) 176-178 (1980).

70. N. A. F. Jaeger and F. Rahmatian, "Integrated optics Pockels cell high-voltage sensor," IEEE Trans. Power Del. 10(1), 127-134, (1995).

71. O. Ogawa, T. Sowa, and S. Ichizono, "A guided-wave optical electric field sensor with improved temperature stability," J. Lightwave Technol. 17(5), 823-830 (1999).

72. H. Wang, R. Zeng, and C. Zhuang, "Thermal variation of electric field sensor bias caused by anisotropy of $\mathrm{LiNbO}_{3}$," Appl. Phys. Lett. 114, 143501 (2019).

73. N. Courjal et al., "Simple production of membrane-based $\mathrm{LiNbO}_{3}$ micro-modulators with integrated tapers," Opt. Lett. 41, 5110-5113 (2016).

74. R. Aydé et al., "Unbiased electro-optic waveguide as a sensitive nuclear magnetic resonance sensor," IEEE Photonics Technol. Lett. 26(12), 1266-1269 (2014). 
75. C. Gutiérrez-Martínez, J. Santos-Aguilar, and A. Morales-Díaz, "On the design of videobandwidth electric field sensing systems using dielectric $\mathrm{LiNbO}_{3}$ electro-optic sensors and optical delays as signal carriers," IEEE Sens. J. 13(11), 4196-4203 (2013).

76. N. Kuwabara et al., "Development and analysis of electric field sensor using $\mathrm{LiNbO}_{3}$ optical modulator," IEEE Trans. Electron. Comput. 34, 391-396 (1992).

77. R. Zeng et al., "Design and application of an integrated electro-optic sensor for intensive electric field measurement," IEEE Trans. Dielectr. Electr. Insul. 18, 312-319 (2011).

78. D. H. Naghski et al., "An integrated photonic Mach-Zehnder interferometer with no electrodes for sensing electric fields," J. Lightwave Technol. 12(6), 1092-1098 (1994).

79. Q. Zhang et al., "Reconfigurable directional coupler in lithium niobate crystal fabricated by three-dimensional femtosecond laser focal field engineering," Photonics Res. 7, 503-507 (2019).

80. T. Meier et al., "Electro-optical E-field sensor with optimised electrode structure," Electron. Lett. 28, 1327-1329 (1992).

81. R. Kobayashi, K. Tajima, and N. Kuwabara, "Optical bias angle control method for electric field sensor using Mach-Zehnder interferometer," Electron. Commun. Jpn. 83(8), 53-61 (2000).

82. J. Qi et al., "Fabrication of polarization-independent single-mode waveguides in lithium niobate crystal with femtosecond laser pulses," Opt. Mater. Express 6, 2554-2559 (2016).

83. J. Zhang et al., "A novel bias angle control system for $\mathrm{LiNbO}_{3}$ photonic sensor using wavelength tuning," IEEE Photonics Technol. Lett. 25(20), 1993-1995 (2013).

84. J. E. Toney et al., "Remote operating point control of Mach-Zehnder interferometer based electric field sensor by power over fiber," in 2nd Int. Workshop Photonics Appl. EM Meas., Zurich, Switzerland, pp. 5-6 (2017).

85. J. E. Toney et al., "Operating point correction for Mach-Zehnder interferometer-based electro-optic E-field sensors," in Opt. Sens. and Sens. Cong. (ES, FTS, HISE, Sensors), OSA Technical Digest, Optical Society of America, SW4C.1 (2019).

86. C. Gutierrez-Martinez and R. Ricardez-Trejo, "Remotely biasing the electro-optic response of an electric field sensing-detection system using LiNbO3 asymmetric Mach-Zehnder optical retarders," Appl. Opt. 57, 9677-9682 (2018).

87. W. Sohler et al., "Integrated optical devices in lithium niobate," Opt. Photonics News 19(1), 24-31 (2008).

88. B. T. Walkenhorst, V. Rodriguez, and J. Toney, "Characterization of a photonics E-field sensor as a near-field probe," in Antenna Meas. Tech. Assoc. Symp., Atlanta, GA, pp. 1-6 (2017).

89. H. Murata, "Millimeter-wave-band electro-optic modulators using antenna-coupled electrodes for microwave photonic applications," J. Lightwave Technol. 38, 5485-5491 (2020).

90. https://www.nanoln.com/.

91. https://www.soitec.com/en/products/smart-cut.

92. M. Levy et al., "Fabrication of single-crystal lithium niobate films by crystal ion slicing," Appl. Phys. Lett. 73, 2293 (1998).

93. M. Xu et al., "High-performance coherent optical modulators based on thin-film lithium niobate platform," Nat. Commun. 11, 3911 (2020).

94. A. Honardoost, K. Abdelsalam, and S. Fathpour, "Rejuvenating a versatile photonic material: thin-film lithium niobate," Laser Photonics Rev. 14(9), 2000088 (2020).

95. D. J. Wilson et al., "Integrated gallium phosphide nonlinear photonics," Nat. Photonics 14, 57-62 (2020).

96. F. Behague et al., "Low-loss BSO photonic waveguides," in Front. Opt./Laser Sci., OSA Technical Digest, Optical Society of America, JW3A.23 (2018).

97. J. E. Ortmann et al., "Epitaxial oxides on glass: a platform for integrated oxide devices," ACS Appl. Nano Mater. 2(12), 7713-7718 (2019).

98. H. Sun et al., "All-dielectric electrooptic sensor based on a polymer microresonator coupled side-polished optical fiber," IEEE Sens. J. 7(4), 515-524 (2007).

99. R. Gibson, R. Selfridge, and S. Schultz, "Electro-optic sensor from high-Q resonance between optical D-fiber and slab waveguide," in Conf. Lasers and Electro-Opt./ 
Quantum Electron. and Laser Sci. Conf. and Photonic Appl. Syst. Technol., OSA Technical Digest (CD), Optical Society of America, CMJ2 (2008).

100. D. Perry et al., "Multiaxis electric field sensing using slab coupled optical sensors," Appl. Opt. 52, 1968-1977 (2013).

101. L. Chen and R. M. Reano, "Compact electric field sensors based on indirect bonding of lithium niobate to silicon microrings," Opt. Express 20, 4032-4038 (2012).

102. Y. Qi and Y. Li, "Integrated lithium niobate photonics," Nanophotonics 9(6), 1287-1320 (2020).

103. R. Wu et al., "Lithium niobate micro-disk resonators of quality factors above $10^{7}$," Opt. Lett. 43, 4116-4119 (2018).

104. https://www.nonlinearmaterials.com.

105. https://www.polariton.ch.

106. I.-C. Benea-Chelmus et al., "Electro-optic interface for ultrasensitive intracavity electric field measurements at microwave and terahertz frequencies," Optica 7, 498-505 (2020).

107. Y. Salamin et al., "Compact and ultra-efficient broadband plasmonic terahertz field detector," Nat. Commun. 10, 5550 (2019).

108. http://creativecommons.org/licenses/by/4.0/.

109. M. Soljačić et al., "Photonic-crystal slow-light enhancement of nonlinear phase sensitivity," J. Opt. Soc. Am. B 19, 2052-2059 (2002).

110. H. Lu et al., "Enhanced electro-optical lithium niobate photonic crystal wire waveguide on a smart-cut thin film," Opt. Express 20, 2974-2981 (2012).

111. N. Courjal et al., "Photonic bandgap properties of lithium niobate," in Ferroelectric Crystals for Photonic Applications, P. Ferraro, S. Grilli, and P. De Natale, Eds., Springer Series in Materials Science, Vol. 91, pp. 313-335, Springer, Berlin, Heidelberg (2014).

112. M. Roussey et al., "Electro-optic effect exaltation on lithium niobate photonic crystals due to slow photons," Appl. Phys. Lett. 89, 241110, (2006).

113. W. Qiu et al., "Guided resonances on lithium niobate for extremely small electric field detection investigated by accurate sensitivity analysis," Opt. Express 24, 20196-20209 (2016).

114. A. Hoblos et al., "Excitation of symmetry protected modes in a lithium niobate membrane photonic crystal for sensing applications," OSA Continuиm 3 (11), 3008-3018 (2020).

115. J. Zhang et al., "An isotropic intense electromagnetic impulse (EMP) sensor using integrated electro-optic (EO) probe," Optik 147, 385-390 (2017).

116. I. C. Benea-Chelmus et al., "Electric field correlation measurements on the electromagnetic vacuum state," Nature 568, 202-206 (2019).

117. A. Mostajabi et al., "Single-sensor source localization using electromagnetic time reversal and deep transfer learning: application to lightning," Sci. Rep. 9, 17372 (2019).

118. L. Wang et al., "Single-shot and single-sensor high/super-resolution microwave imaging based on metasurface," Sci. Rep. 6, 26959 (2016).

Florent Behague received his master's degree in nanosciences from the University of Burgundy, Dijon, France, in 2016. Since 2017, he has been earning his PhD in integrated optics and photonics at Franche-Comte University, Besançon, France. His research interests include the development of ultra-thin integrated polarization control devices and polarization-independent EO waveguides for $E$-field sensors.

Venancio Calero studied his bachelor's degree in telecommunication engineering at the Miguel Hernandez University. Since then, he has focused on optics and nanosciences along his experience as a research assistant at the Optical Materials Chair (Miguel Hernandez University) and the Nano-Materials Chair (Dresden University of Technology). He holds his PhD in optics and photonics at Franche-Comté University for his works on ferroelectric materials-based photonic devices at the FEMTO-ST Institute. Currently, he is a research engineer at Seagate Technology exploring further nanophotonics.

Antoine Coste graduated from the Ecole Normale Supérieur de Lyon in 2015. He received his master's degree in nanophysics in 2016 from Paris-Saclay University and his $\mathrm{PhD}$ in plasmonic and quantum optics in 2019 from Paris-Saclay University. Since 2020, he has been working as a 
research engineer at the FEMTO-ST Institute, Besançon, France. He works on the production of electro-optic sensors in partnership at Kapteos SAS.

Adrien Godet received his MS degree in physics from the Université de Rennes, France, in 2015 and his $\mathrm{PhD}$ in optics and photonics from the FEMTO-ST Institute, Universite de FrancheComté, Besançon, France, in 2018. His thesis research was on nanofiber-optic sensors. Since 2019, he has been a postdoctoral research engineer at the FEMTO-ST Institute to go on his thesis works, and he recently worked on the nanophotonics domain with the development of electrooptic probes in lithium niobate.

Miguel Suarez obtained his MS degree in physics from the Universidad Industrial de Santander, Bucaramanga, Colombia, in 2001 and his $\mathrm{PhD}$ in engineering science from Franche-Comté University, Besançon, France, in 2006. After four postdoctoral positions in different research centers between 2006 and 2015, he integrated the Optics Department of the FEMTO-ST Institute, Besançon, France as a research engineer. His expertise is based on nanomanufacturing processes and the development of optical setups for automatic characterization.

Gwenaël Gaborit received his $\mathrm{PhD}$ in physics from the University of Savoie in 2005. Since 2006, he has been an associate professor at IMEP-LAHC Laboratory. He is also a co-founder and a chief science officer of Kapteos SAS. He passed the French accreditation to supervise research (HDR) in 2014. His main research interest focuses on electro-optic sensors dedicated to noninvasive electric field characterization. He has co-authored more than 100 journal papers and international conference proceedings. He holds four patents.

Lionel Duvillaret has been an associated professor at Savoy University up to 2005, after a $\mathrm{PhD}$ carried out at Paris-Sud in 1994. Then he became a professor at Grenoble Polytechnic Institute. He founded Kapteos SAS in 2009 and he is currently its CEO. He is the author or co-author of dozens of patents and more than 190 papers and international conferences in the field of $E$-field measurements via electro-optic technology, THz time-domain spectroscopy, and electromagnetic bandgap devices.

Fadi I. Baida is a professor at the University of Franche-Comte and a head of the Nano-Optics Team. His research focuses on the modeling of the matter-light interaction at the nanometer scale including near-field imaging, metamaterials, nanoantennas, optical forces, and photonic crystals. He has acquired a profound expertise in the implementation and adaptation of the finite-difference-time-domain method to the field of nanooptics in addition to analytical methods such as Rayleigh perturbation method and modal methods.

Maria-Pilar Bernal received her PhD in physics from the University of Zaragoza, Spain, in 1998. From 1994 to 1998, she participated in a joint study between the University of Zaragoza and the IBM Almaden Research Center, where she worked in the field of holographic data storage. Subsequently, she was a research assistant on scanning near-field optical microscopy at the Swiss Federal Institute of Technology, Lausanne, Switzerland. She is a Directeur de Recherche $\mathrm{CNRS}$ at the FEMTO-ST Institute, Besançon, France, where she works in $\mathrm{LiNbO}_{3}$ nanophotonics. To date, she has published more than 100 articles with over 2800 citations.

Nadège Courjal obtained her $\mathrm{PhD}$ in engineering science from Besançon University in 2002. After 2 years of research on electro-optic photonic crystals, she became an associate professor at the FEMTO-ST Institute in 2004. Her research activities are oriented toward the development of low-power-consuming electro-optic components. She is currently an in-charge of a technological platform within the ActPhast4 European network and a co-founder of a Joint Laboratory at Kapteos SAS for the development of minimally invasive EO sensors. 